Article

\title{
Energy-Efficient Forest Fire Prediction Model Based on Two-Stage Adaptive Duty-Cycled Hybrid X-MAC Protocol
}

\author{
Jin-Gu Kang ${ }^{\mathbb{D}}$, Dong-Woo Lim and Jin-Woo Jung * \\ Department of Computer Science and Engineering, Dongguk University, Seoul 04620, Korea; \\ kanggu12@dongguk.edu (J.-G.K.); aehddn@gmail.com (D.-W.L.) \\ * Correspondence: jwjung@dongguk.edu; Tel.: +82-2-2260-3812
}

Received: 7 August 2018; Accepted: 3 September 2018; Published: 5 September 2018

\begin{abstract}
This paper proposes an adaptive duty-cycled hybrid X-MAC (ADX-MAC) protocol for energy-efficient forest fire prediction. The Asynchronous sensor network protocol, X-MAC protocol, acquires additional environmental status details from each forest fire monitoring sensor for a given period, and then changes the duty-cycle sleep interval to efficiently calculate forest fire occurrence risk according to the environment. Performance was verified experimentally, and the proposed ADX-MAC protocol improved throughput by $19 \%$ and was $24 \%$ more energy efficient compared to the X-MAC protocol. The duty-cycle was shortened as forest fire probability increased, ensuring forest fires were detected at faster cycle rate.
\end{abstract}

Keywords: forest fire; prediction model; energy efficient; sensors; Wireless Sensor Network; X-MAC; hybrid; adaptive; duty-cycle; protocol

\section{Introduction}

Forest fires spread rapidly according to topography and mountain slope if they cannot evolve at an early stage due to their nature. In particular, forest fires generated during the day spread more widely, cannot evolve, and are transferred to deeper mountain areas.

Figure 1 shows forest fire occurrence in South Korea. An average of 340,000 people die every year due to forest fires worldwide [1], and average yearly damage caused by forest fires over the last 10 years was approximately 10 million US Dollars [2]. Destroyed forests, damaged buildings, and human injuries and casualties due to forest fires should all be avoided. Forest fire watching, administered separately by local governments in South Korea, has a human cost of approximately 0.5 million US Dollar for each local government [1]. The goal of this paper was to reduce this damage, recovery, and maintenance costs without increasing forest fire risk.

The average forest area in South Korea is about 6 million hectares [2]. When the proposed protocol is applied to a sensor capable of covering a $300 \mathrm{~m}$ range, the number of required sensors is about 1000 . It does not cover all areas of the forest but is the central area and the passenger path where forest fires can intensify. The areas that are actually in contact with the surface and the summits are very unlikely to develop into forest fires, and the fire is naturally turned off. When the price of one sensor is set at 200 US Dollar, it costs about 2.2 million US Dollar for maintenance costs, such as sensor cost and battery replacement. The cost of the sensor using the protocol proposed in this paper is about $1 / 4$ times lower than the existing cost, and the $24 \mathrm{~h}$ surveillance is cost effective. There is an advantage in that it is possible. The biggest advantage of being able to keep watch $24 \mathrm{~h}$ is that you can completely prevent forest fires. In addition, even if the existing labor cost is replaced with a sensor, the maintenance cost of the battery also exists. When sensors are used, energy-efficiency is the most considered part. 


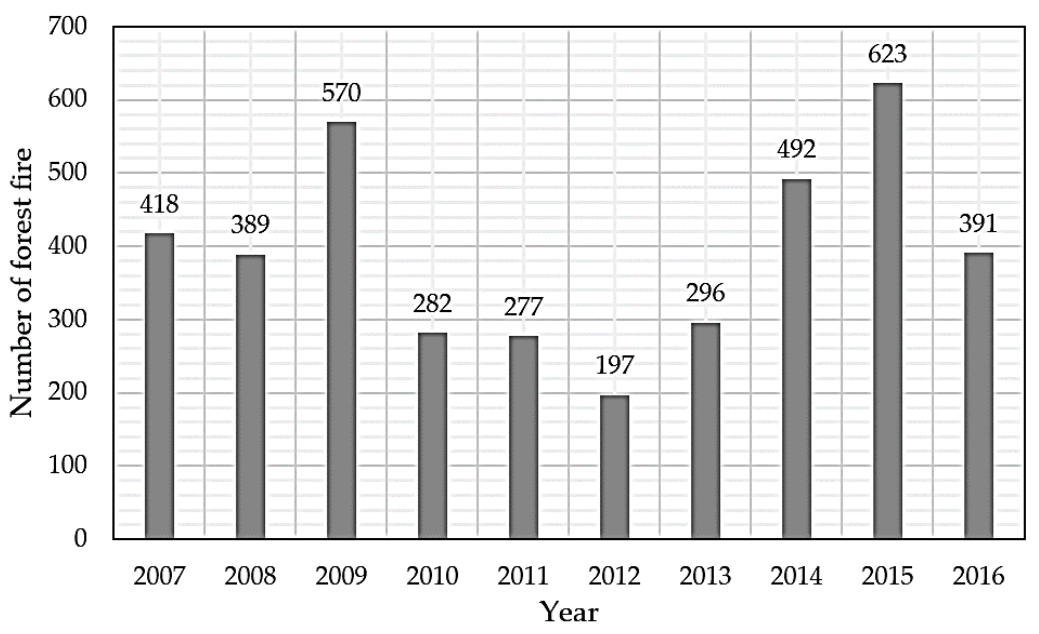

Figure 1. South Korea total yearly forest fires.

Synchronous and asynchronous medium access control (MAC) protocols have been proposed to reduce energy consumption, e.g., such as idle energy [3,4], which operates on the basis of a duty-cycle. Traffic increases as the number of connected devices increases, but node duty-cycles remain unchanged. Consequently, opportunities to send data decrease and throughput decreases, as listening interval durations increase. In particular, a forest fire's sudden and natural escalation means there is small traffic by the initial sensors that very rapidly increases as the fire develops.

Therefore, an adaptive duty-cycled hybrid X-MAC (ADX-MAC) protocol based on X-MAC protocol [5] is proposed to implement an energy efficient forest fire prediction system that more efficiently reduces energy consumption during sensor waiting times and speeds up data transmission between nodes.

\section{Forest Fire Prediction}

The forest fire prediction model [6] is selected by data used, including nationwide forest fire data for 25 years (1991-2015), consisting of 10,560 observations and other details regarding forest fire areas and characteristics. Table 1 summarizes the variables included in the forest fire damage records $[1,6,7]$.

Table 1. Fire damage data from the forest fire prediction model.

\begin{tabular}{clc}
\hline Field & \multicolumn{1}{c}{ Description } & Example \\
\hline Year_No & Annual number (starting from 1 every year) & 1 \\
Month_No & Annual number (starting from 1 every year) & 1 \\
F_Year & Year of occurrence & 1991 \\
F_Month & Month of occurrence & 1 \\
F_Day & Day of occurrence & 13 \\
F_Time & Time of occurrence & $13: 40$ \\
F_Week & Date of occurrence & SUN \\
E_Year & Year of extinguish & 1991 \\
E_Month & Month of extinguish & 1 \\
E_Day & Day of extinguish & 14 \\
E_Time & Completion time of extinguish & $14: 55$ \\
R_Time & Extinguish time & $19: 30$ \\
Loc_Type & Station 1 (with errors) & Ulsan \\
G_Offices & Station 2 (with errors) & Ulsan \\
Loc_SiDo & City (Si), State (Do) & Ulsan \\
Loc_Gung & Street 1 (Gun, Gu) & Buk \\
Loc_Dong & Street 2 (Dong) & \\
Loc_Ri & Street 3 (Lee) &
\end{tabular}


Table 1. Cont

\begin{tabular}{clc}
\hline Field & \multicolumn{1}{c}{ Description } & Example \\
\hline PNU_Nam & Other addresses & Mt. 79-1 \\
Owner_Ty & Ownership type & \\
F_Type & Types of forest fires & \\
F_Cause & Cigarette burning, trash incineration, building fire transfer, arson, & Construction site, bonfire \\
(11 items) & field incineration, mistake by children, climbing, in operation, <rest> & 14 \\
D_Area & Damage area & 0 \\
D_AreaU & Additional damage area & 14 \\
D_AreaT & Total damage area & 1260 \\
D_Amount & Amount of damage & 2.8 \\
Temprt & Temperature & NNW \\
Wind_Dir & Wind direction & 6 \\
Wind_Sped & Wind speed & 26 \\
Humidity & Relative humidity & 6 \\
Lapse_Day & Elapsed days since 5 mm or more rainfall & 1.8 \\
R_Amount & Rainfall & 416976.8 \\
Coord_X & X coordinate for location & 229115.2 \\
Coord_Y & Y coordinate for location & Ulsan Metropolitan City, Bukgu \\
Address & City (Si), State (Do) (Including special metropolitan city) & \\
\hline
\end{tabular}

\subsection{Prediction Model Weights}

The prediction model used the weather and location information relevant to the forest fire occurrence and parameters for human accessibility. It employed spatial kriging to include meteorological information, distance from dense populations to the fire location, number of trails in the lattice, and altitude data to generate accessibility parameters. The available data was modified to correspond to a $5 \mathrm{~km}$ grid. The experiment selected logistic regression as the forest fire prediction model and it was reflected in the monthly effect model using variable numbers for refinement. The model achieved $84.8 \%$ predicted rate, which was very significant.

Main forest fire contributing factors include temperature, wind speed, humidity, and rainfall. Table 2 shows the correlations for these climate factors according to the forest fire prediction model.

Table 2. Correlation between forest fire frequency and climate factors.

\begin{tabular}{ccc}
\hline Factor & Correlation $\left(\boldsymbol{w}_{\boldsymbol{X}}\right)$ & Test Statistic \\
\hline Temperature $(T P)$ & -0.043 & -0.7429 \\
Wind speed $(W S)$ & 0.304 & 5.5086 \\
Humidity $(H)$ & -0.198 & -3.4870 \\
Rainfall $(R)$ & -0.105 & -1.8226 \\
\hline
\end{tabular}

The correlation between the number of forest fires and temperature (TP) is almost insignificant, whereas wind speed $(W S)$, humidity $(H)$, and rainfall $(R)$ correlations are all significant at this level. Thus, forest fire probability increases when wind speed is high, humidity is low, and rainfall is small.

Forest fire risk in terms of weighted climate factors can be estimated as:

$$
f_{\text {risk }}(t)=\left|\frac{\Delta_{W S}(t)}{\Delta_{T P}(t) \Delta_{H}(t) \Delta_{R}(t)}\right|
$$

where

$$
\Delta_{X}(t)=\left\{\begin{aligned}
w_{X} \frac{X(t)-X_{\min }}{X_{\max }-X_{\min }}, & X(t)>X_{\min } \\
1, & X(t) \leq X_{\min }
\end{aligned}\right.
$$

where $X_{t}$ is the measured climate factor over time frame $t$ with $X_{\max }$ and $X_{\min }$ maximum and minimum, respectively, over the period; and $w_{X}$ is the correlation coefficient from Table 1, i.e., the weight for each factor. Therefore, each $\Delta_{X}$ is a weighted ratio to the uniform average change of all factors. 


\subsection{Forest Fire Risk Analysis from Test Statistics and Weights}

Tables 3 and 4 show climate factors for Gangneung and Surak Mountains in South Korea for January to October 2017 [2,7]. Stair function test statistics included 0-5, considering the minimum and maximum for each climate factor in the mountain range. Figure 2 shows the data from Tables 3 and 4 graphically.

Table 3. Forest fire risk analysis for Mt. Gangneung, 2017.

\begin{tabular}{ccccccccccc}
\hline Factor & January & February & March & April & May & June & July & August & September & October \\
\hline \multicolumn{10}{c}{ Measured climate factor } \\
\hline$W S(\mathrm{~m} / \mathrm{s})$ & 3 & 3.1 & 2.4 & 2.8 & 23 & 1.9 & 2 & 1.6 & 2.3 & 1.9 \\
$H(\%)$ & 43.3 & 38.2 & 48.5 & 44.3 & 29 & 61.4 & 67.9 & 74.4 & 61.2 & 67.2 \\
$R(\mathrm{~mm})$ & 48.5 & 3.5 & 48.4 & 39.8 & 0 & 27.2 & 238.1 & 444.1 & 45.7 & 114.5 \\
$T P\left({ }^{\circ} \mathrm{C}\right)$ & 1.5 & 3.5 & 7.1 & 15.4 & 17.9 & 21.2 & 26.8 & 24.3 & 21.3 & 15.2 \\
\hline \multicolumn{10}{c}{ Stair function test statistic } \\
\hline$W S(\mathrm{~m} / \mathrm{s})$ & 0 & 0 & 0 & 0 & 4 & 0 & 0 & 0 & 0 & 0 \\
$H(\%)$ & 2 & 3 & 2 & 2 & 4 & 0 & 0 & 0 & 0 & 0 \\
$R(\mathrm{~mm})$ & 4 & 4 & 4 & 4 & 4 & 4 & 2 & 0 & 4 & 3 \\
$T P\left({ }^{\circ} \mathrm{C}\right)$ & 0 & 0 & 0 & 0 & 0 & 1 & 2 & 1 & 1 & 0 \\
\hline \multicolumn{8}{c}{ Forest fire prediction model } \\
\hline$f_{\text {risk }}$ & 0 & 0 & 0.0001 & 0.5610 & 0 & 0.0003 & 0.0003 & 0.0001 & 0.0001 \\
\hline
\end{tabular}

The stair function is to compute each climatic factor as a single graph regardless of the unit dimension, and it is divided into stages according to the numerical value of each climatic factor. WS is more than 5 to $11 \mathrm{~m} / \mathrm{s}, H$ is 60 to $30 \%, R$ is 400 to $100 \mathrm{~mm}$, and $T P$ is 20 to $35^{\circ} \mathrm{C}$. The figures set for each step show the average degree of climate change in the forest area of South Korea.

Table 4. Risk Analysis of Forest Fire in Mt. Surak, 2017.

\begin{tabular}{ccccccccccc}
\hline Factor & January & February & March & April & May & June & July & August & September & October \\
\hline \multicolumn{10}{c}{ Measured climate factor } \\
\hline$W S(\mathrm{~m} / \mathrm{s})$ & 2.3 & 2.5 & 2.4 & 2.6 & 2.4 & 5 & 2 & 2.3 & 2 & 1.9 \\
$H(\%)$ & 54.6 & 53.7 & 48.1 & 52.4 & 52.2 & 43 & 77.2 & 70.8 & 60.9 & 56.3 \\
$R(\mathrm{~mm})$ & 0 & 0 & 0 & 0 & 0 & 0 & 0 & 0 & 0 & 0 \\
$T P\left({ }^{\circ} \mathrm{C}\right)$ & -1.8 & -0.2 & 6.3 & 13.9 & 19.5 & 23.3 & 26.9 & 25.9 & 22.1 & 16.4 \\
\hline \multicolumn{10}{c}{ Stair function test statistic } \\
\hline$W S(\mathrm{~m} / \mathrm{s})$ & 0 & 0 & 0 & 0 & 0 & 1 & 0 & 0 & 0 & 0 \\
$H(\%)$ & 1 & 1 & 2 & 1 & 1 & 2 & 0 & 0 & 0 & 1 \\
$R(\mathrm{~mm})$ & 0 & 0 & 0 & 0 & 0 & 4 & 0 & 0 & 0 & 0 \\
$T P\left({ }^{\circ} \mathrm{C}\right)$ & 0 & 0 & 0 & 0 & 0 & 1 & 2 & 2 & 1 & 0 \\
\hline \multicolumn{10}{c}{ Forest fire prediction model } \\
\hline$f_{\text {risk }}$ & 0.0006 & 0.0064 & 0.0469 & 0.0670 & 0.08061 .102 & 0.0254 & 0.0385 & 0.0377 & 0.0332 \\
\hline
\end{tabular}

The Mt. Gangneung forest fire, 8 May 2017, had $f_{\text {risk }}=0.5610$, i.e., very high compared to normal values, and the Mt. Surak forest fire, 1 June 2017, had $f_{\text {risk }}=1.102$, i.e., extremely high. Thus, model estimates are reasonable, and hence $f_{\text {risk }}$ was adopted as a core parameter for the proposed protocol. However, $f_{\text {risk }}$ does not specify that a forest fire will occur, but only that the probability of occurrence is high. Figure 2 shows that $f_{\text {risk }}$ is most influenced by WS among the climate factors, but there are some factors considered by other climate factors. 


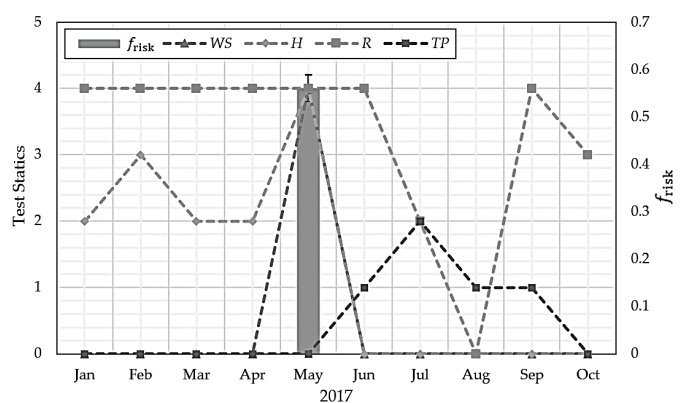

(a)

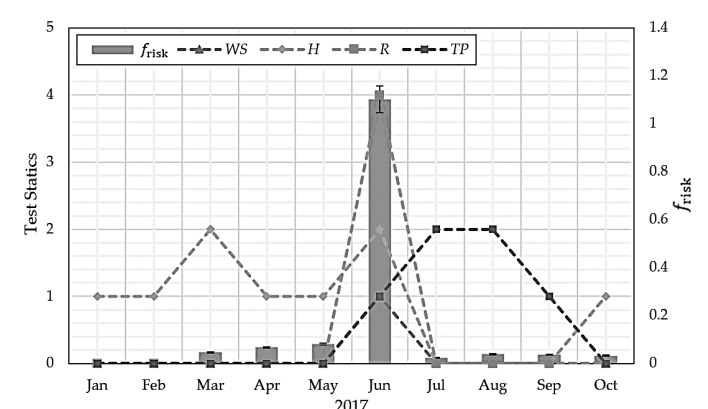

(b)

Figure 2. Test statistic variation with duty-cycle length for (a) Mt. Gangneung and (b) Mt. Surak.

\section{Energy Efficient Forest Fire Prediction Protocol}

\subsection{Duty-cycle Wireless Sensor Networks}

Figure 3 shows the asynchronous sensor network protocol, i.e., the X-MAC protocol [4], that was proposed to solve the Berkley MAC (B-MAC) low power listening preamble overhearing problem [8]. X-MAC uses the basic B-MAC methods, but in contrast to the long B-MAC preamble, it transmits the node address as a short preamble followed by a listening to ensure they will receive it.

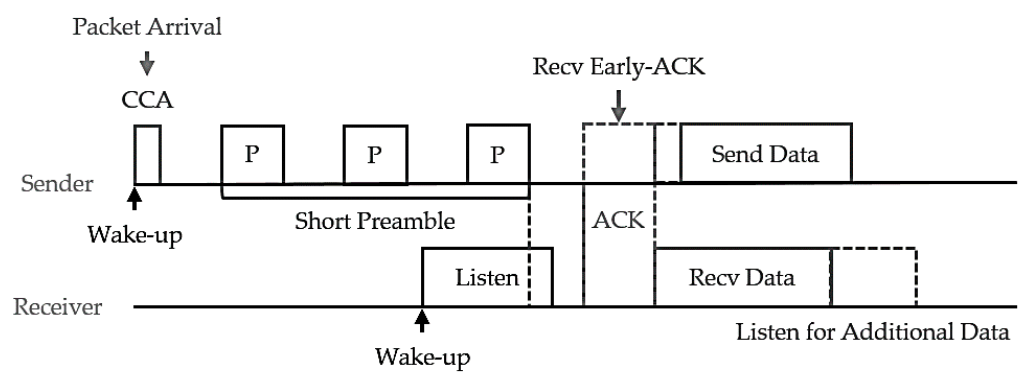

Figure 3. X-MAC protocol.

A receiver node receives the preamble containing its own address value and sends an Early-ACK to a sender node to inform it of preamble reception. When the sender node receives the Early-ACK, it stops transmitting the preamble and immediately transmits the data, thereby transferring data between the two nodes. If the receiver node does not detect its address in the preamble, it immediately returns to idle mode. Thus, control packet overhead is significantly reduced compared to B-MAC, and returning to idle mode effectively reduces overhearing.

Figure 4 shows the proposed adaptive duty-cycled hybrid X-MAC (ADX-MAC) protocol [9].

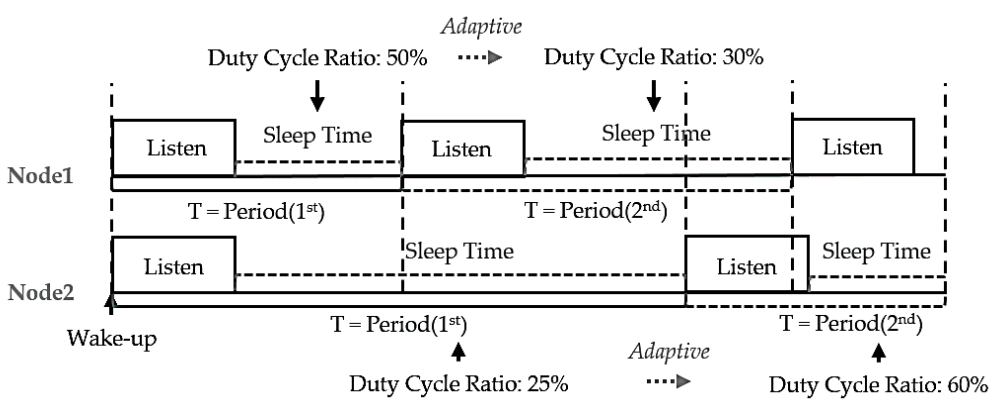

Figure 4. Proposed adaptive duty-cycled hybrid X-MAC protocol. 
Normal X-MAC has a constant duty-cycle, whereas the proposed ADX-MAC duty-cycle, and hence sleep interval, changes according to the current situation. This has a similar effect to contention window (CW) [10] for preventing collision, hence collision probability within a given time (slot) is significantly reduced because the node duty is staggered without Backoff [11], reducing unnecessary delay. For example, when the number of packets in each node's queue is less than the number of packets in the queue, the sleep cycle is increased by decreasing the duty-cycle, reducing energy consumption. On the other hand, when the number of packets increases, particularly when the queue maximum is reached, the duty-cycle is increased, but as little as possible, shortening the sleep interval, and traffic can be quickly resolved. Adapting the duty-cycle to the traffic situation improves energy efficiency compared to throughput by adjusting the unnecessary sleep interval and the time when the node wakes up.

\subsection{Proposed Adaptive Duty-cycled Hybrid X-MAC}

The proposed ADX-MAC condition for controlling duty-cycle length is the number of packets in the queue and $f_{\text {risk }}$ value.

Figure 5 shows that the proposed ADX-MAC header components are almost identical to the X-MAC. However, when the sender sends data to the receiver and the receiver and sender have a similar cycle, the sender's duty-cycle length is included in the header content and may also be included in the Early-ACK or ACK transmission, in contrast to the usual MAC header.

\begin{tabular}{|c|c|c|c|c|c|}
\hline 2bytes & 1byte & $0-20$ bytes & 1byte & Variable & 2bytes \\
\hline $\begin{array}{l}\text { Frame } \\
\text { Control }\end{array}$ & $\begin{array}{c}\text { Seq. } \\
\text { Number }\end{array}$ & Address & $\begin{array}{c}\text { Adjusted } \\
\text { Duty- } \\
\text { Cycle Len. }\end{array}$ & Payload & $\begin{array}{c}\text { Frame } \\
\text { Check } \\
\text { Seq. }\end{array}$ \\
\hline
\end{tabular}

Figure 5. MAC Header (Data Unit) of ADX-MAC.

Figure 6 shows the proposed ADX-MAC algorithm. When the sender wakes up, it first checks the node queue status too determine if there is data to be sent, and if so, operates identically to the X-MAC algorithm, except that X-MAC checks channel status immediately, whereas ADX-MAC first checks duty-cycle length to determine if change (adjust) is required. If duty-cycle length needs to change, this is scheduled (reserved) for the next cycle. If no change is required, the process proceeds to the next step.

The ratio of the number of packets in the queue to the total maximum queue length determines the duty-cycle length for the queue:

$$
Q_{\text {ratio }}(t)=Q(t) / Q_{\max }
$$

where $Q(t)$ is the number of packets queued at current time, $t$; and $Q_{\max }$ is the maximum number of packets that can be queued.

It is then decided whether to change duty-cycle length according to $f_{\text {risk. }}$. If there is data to send immediately, then any duty-cycle change is scheduled for the next cycle. If no change is required the node checks channel status and then transmits the short preamble until it receives Early-ACK. After the data is transmitted the node switches to sleep state.

The increase rate per unit time is:

$$
f_{\text {incr }}(t)=\left\{\begin{aligned}
f_{\text {risk }}(t) / f_{\text {risk }}\left(t_{0}\right), & f_{\text {risk }}\left(t_{0}\right) \neq 0 \\
1, & f_{\text {risk }}\left(t_{0}\right)=0 \\
0, & t=0
\end{aligned}\right.
$$

where $t_{0}$ is the previous time unit and $t$ is the current time unit. 
This is divided into two stages because forest fires develop extremely quickly, and traffic congestion can occur during a one-time unit. Therefore, $Q(t)$ measure channel congestion and $f_{\text {risk }}$ predict and respond quickly to the situation before it occurs, sending emergency data without interruption as the forest fire develops. Thus, combining Equations (3) and (4), the new adaptive duty-cycle is:

$$
T_{\text {adp }}(t)=\left\{\begin{aligned}
1-\left(f_{\text {incr }}(t)+Q_{\text {ratio }}(t)\right)+f_{\text {incr }}(t) Q_{\text {ratio }}(t), & f_{\text {incr }}(t) \leq f_{\text {thd }} \\
1-\left(f_{\text {thd }}+Q_{\text {ratio }}(t)\right)+f_{\text {thd }} Q_{\text {ratio }}(t), & f_{\text {incr }}(t)>f_{\text {thd }}
\end{aligned}\right.
$$

where $f_{\text {thd }}$ is an arbitrary threshold to limit $f_{\text {incr. }}$. Thus, the applied duty-cycle length is:

$$
T(t)=\left\{\begin{aligned}
T_{\mathrm{adp}}(t) T_{0}, & T_{\mathrm{adp}}(t) T_{0} \geq T_{\text {listen }} \\
T_{\text {listen }}, & T_{\mathrm{adp}}(t) T_{0}<T_{\text {listen }}
\end{aligned}\right.
$$

which can grow very large for severe climate factor changes. Although $T$ depends on values obtained from Equations (3) and (4), $T$ cannot be smaller than the listen time, $T_{\text {listen, }}$ if calculated $T<T_{\text {listen }}$ $T \rightarrow T_{\text {listen }}$, and hence duty-cycle ratio $=100 \%$.

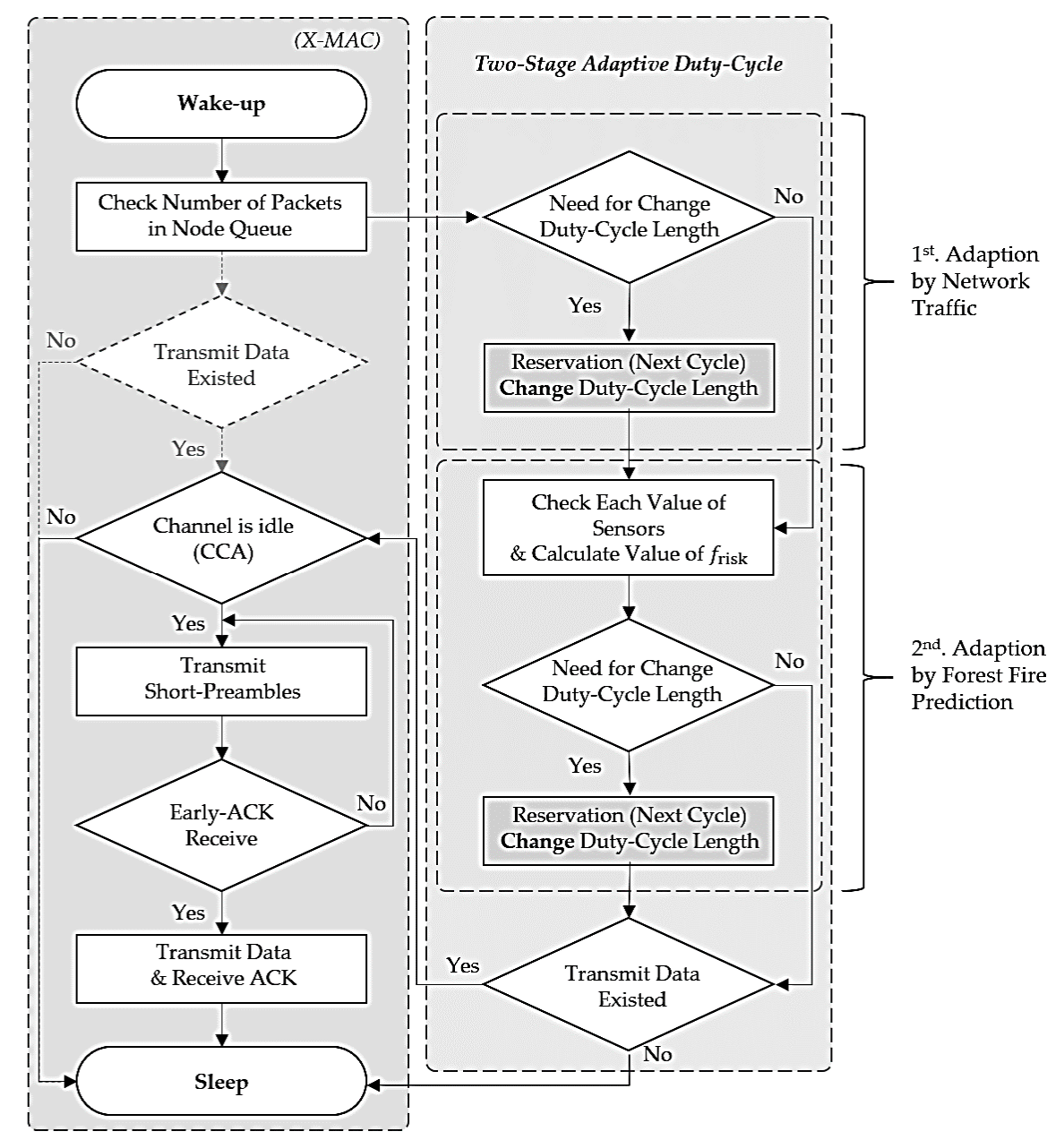

Figure 6. Proposed ADX-MAC algorithm for forest fire.

\section{Experimental Results}

The first experiment compares the throughput and energy consumption with X-MAC to confirm the performance of the proposed protocol, ADX-MAC. However, the number of nodes in this experiment is limited to 5 to 60 . The reason for this is that, since the characteristics of the sensor 
MAC protocol, the collision rate is close to $99 \%$ as long as the CW is not significantly larger than 60 since the performance is significantly lowered. That is, it is the same as the case where all the nodes simultaneously send data during the unit interval in the saturation situation. Therefore, it is possible to confirm the difference in performance between two protocols in a real environment even with this experiment alone. The simulator used in this experiment was a proven NS-2 based simulator [12,13].

Verification in the actual forest fire situation is the most accurate, but it is difficult to generate forest fires artificially. The condition of spontaneous ignition is due to the combination of various climatic factors. Of course, social issues are also included. Therefore, in the second experiment for the forest fire prediction model, each climate factor was measured through an Arduino sensor for a certain unit time. And we confirmed that these measured values matched the duty-cycle length properly.

\subsection{Comparison with $X-M A C$}

Table 5 shows the simulation environment employed. The simulation assumed the same environment and topography for both models, in particular, node distances were the same, packet generation used the constant bit rate method, and the network was always unsaturated.

Table 5. Network topology and simulation environment.

\begin{tabular}{cccc}
\hline Parameter & Value & Parameter & Value \\
\hline Bandwidth & $250 \mathrm{Kbps}$ & Node queue size & 10 \\
Sensing range & $250 \mathrm{~m}$ & Packet (frame) size & 50 bytes \\
Data transmit rate & $5 \mathrm{~ms}$ & Transmit power & $86.2 \mathrm{~mW}$ \\
Wake-up time & $15 \mathrm{~ms}$ & Idle power & $52.2 \mathrm{~mW}$ \\
ACK transmit time & $1 \mathrm{~ms}$ & Receive power & $96.6 \mathrm{~mW}$ \\
SP transmit time & $3 \mathrm{~ms}$ & Sleep power & $0.0183 \mathrm{~mW}$ \\
Initial duty-cycle length & $100 \mathrm{~ms}$ & & \\
\hline
\end{tabular}

Throughput was calculated as

$$
T H R=\frac{C_{\text {ack }} P K T_{\text {size }}}{t_{\text {stop }}-t_{\text {start }}},
$$

where $C_{\text {ack }}$ is the number of ACKs received by the sender until the end of the simulation; $P K T_{\text {size }}$ is the packet size; and $t_{\text {stop }}$ and $t_{\text {start }}$ indicate the time the simulation ended and started, respectively.

Energy consumption was calculated as:

$$
E=\frac{P W R_{\text {use }}}{N\left(t_{\text {stop }}-t_{\text {start }}\right)}
$$

where $P W R_{\text {use }}$ is the power used until the end of the simulation, and $N$ is the total number of nodes.

This experiment did not consider $f_{\text {risk, }}$, because there would be little throughput effect if this was included. Figure 7 compares ADX-MAC and X-MAC throughput. Efficiency is similar up to nodes $=10$, but ADX-MAC has an average efficiency approximately $24 \%$ higher than X-MAC once nodes $=15$, and this advantage persists up to quite large networks.

Figure 8 compares ADX-MAC and X-MAC energy consumption. Average ADX-MAC efficiency was approximately $18 \%$ lower than that of X-MAC up to nodes $=25$, which then extended to $24 \%$ lower. This result is due to the fact that ADX-MAC performs transmission about $1 / 4$ the rate of X-MAC as the number of nodes increases.

Figure 9 compares ADX-MAC and X-MAC energy consumption per byte, i.e., the average amount of energy consumed to transmit one byte until the simulation ended, calculated as:

$$
E_{\text {byte }}=\frac{P W R_{\text {use }}}{C_{\text {ack }} P K T_{\text {size }}} .
$$


The amount of energy consumed per byte can be said to be always good because the ADX-MAC consumes less than the X-MAC over the number of intervals of all nodes. Although the efficiency of ADX-MAC is about $28 \%$ higher than that of X-MAC until the number of nodes is 35 , the efficiency drops rapidly after that. However, the average number of nodes is about $17 \%$ in the range of 40 to 60 . In conclusion, it shows about $24 \%$ efficiency in the all number of nodes.

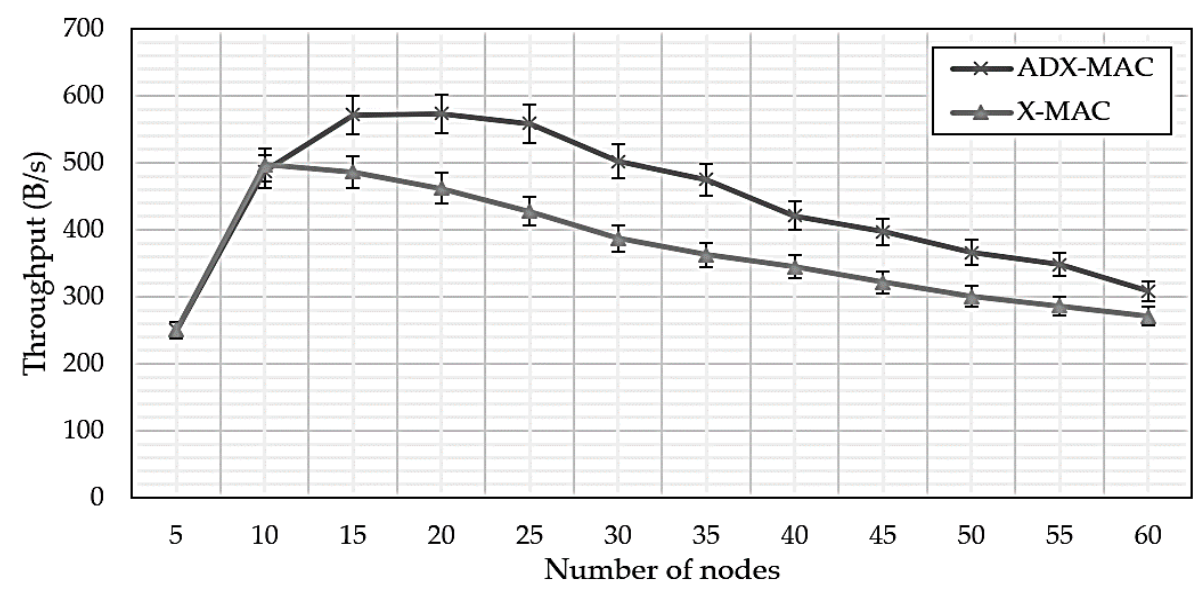

Figure 7. ADX-MAC and X-MAC throughput.

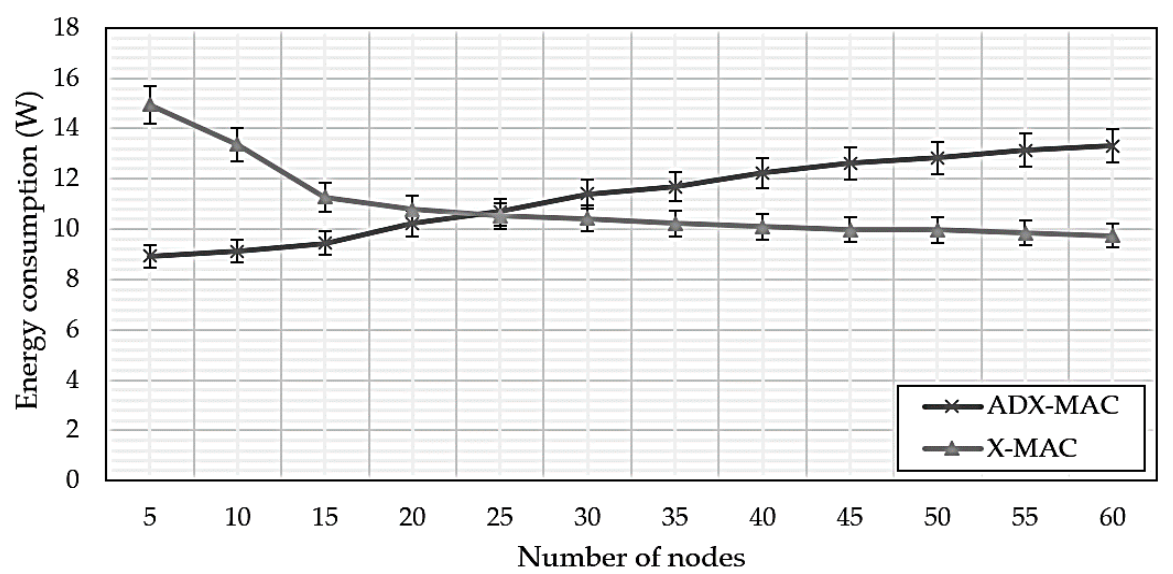

Figure 8. ADX-MAC and X-MAC energy consumption.

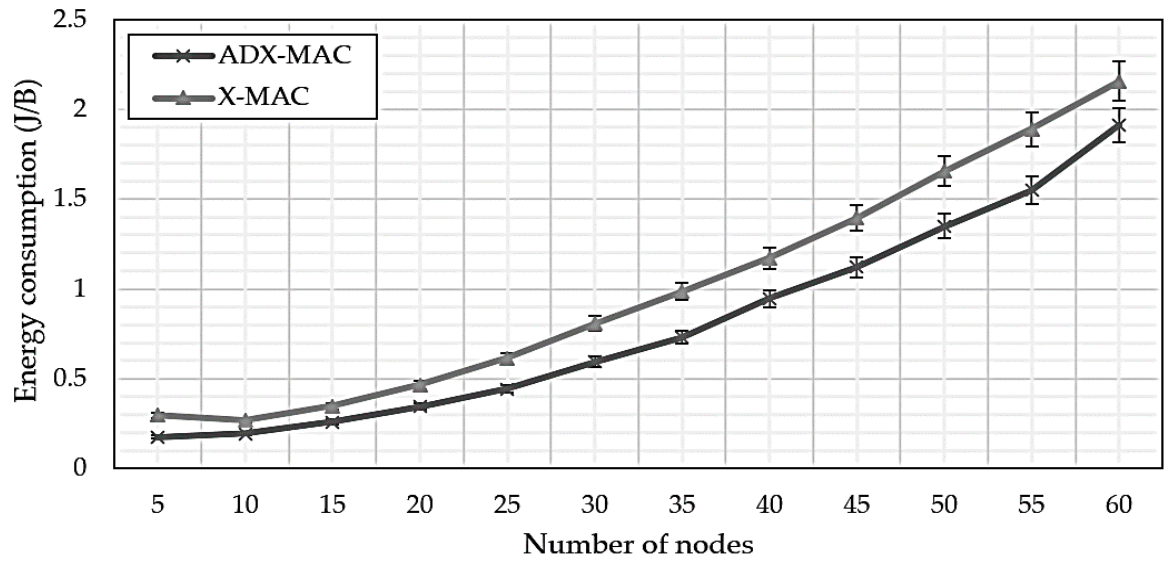

Figure 9. ADX-MAC and X-MAC energy consumption for an individual byte transmitted until the simulation completed. 


\subsection{Adaptive Duty-Cycle Length Due to Climatic Factors}

This experiment investigated how $T$ varied with measured climate factor values. Network setup and parameters were the same as in Section 4.1, but this experiment did not consider the state of the queue, similar to the treatment for $f_{\text {risk }}$ in Section 4.1 .

Figure 10 shows the $T$ changes for each climate factor, considering only the factor of interest to be changing, whereas Figure 11 considers all climatic factors. High $f_{\text {risk }}$ derived from the forest fire prediction model means forest fire probability is high, hence $T$ is shortened, and sensing is performed at a shorter cycle per unit time.

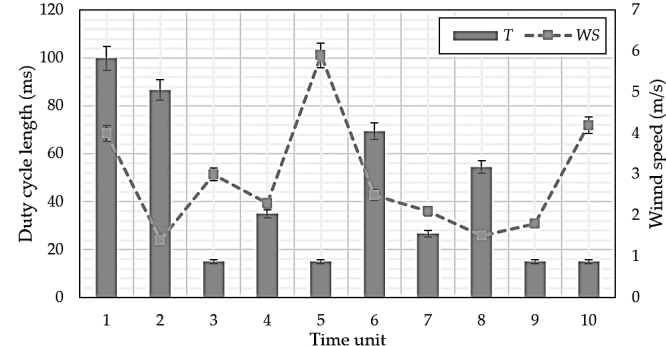

(a)

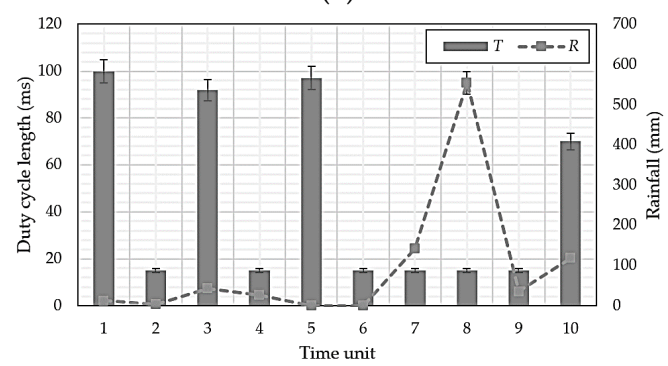

(c)

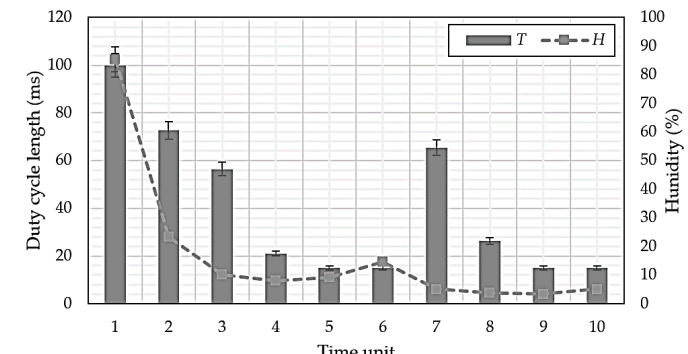

(b)

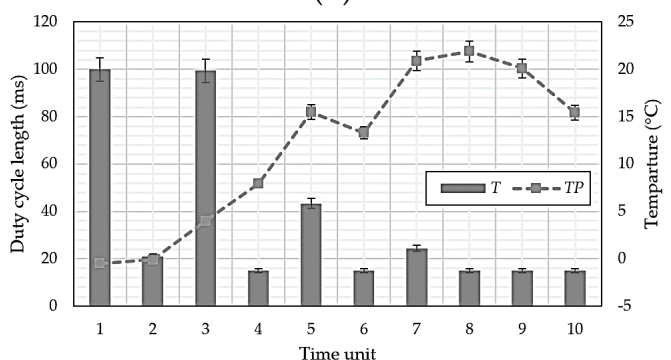

(d)

Figure 10. Duty-cycle length, $T$, changes for climate factor changes (a) wind speed; (b) humidity; (c) rainfall, and (d) temperature.

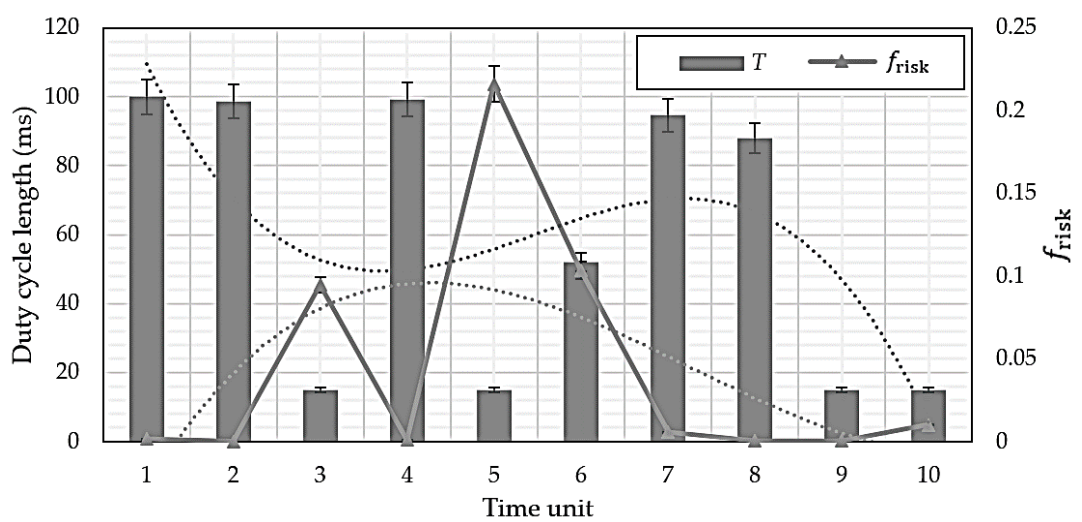

Figure 11. Change in $f_{\text {risk }}$ as duty-cycle length, $T$, changes.

\section{Conclusions}

This paper proposed a forest fire prediction method, i.e., the adaptive duty-cycled hybrid X-MAC (ADX-MAC) protocol, which is based on sensor data to reduce human and economic costs using Wireless Sensor Network (WSN) MAC protocol. The key factors of the proposed method were to adapt duty-cycle length through two stages with different factors. The two stages addressed that forest fires occur very quickly and significant sensor data traffic congestion can occur during a single time unit. 
The first stage factor is $f_{\text {risk, }}$, derived from the forest fire prediction model, and the second stage factor was the number of packets in the node queue. Thus, ADX-MAC could adjust $T$ when considering both the traffic situation and forest fire probability.

The proposed approach was verified experimentally by comparing it with X-MAC, and confirmed that ADX-MAC average throughput and energy efficiency were approximately $19 \%$ and $24 \%$ superior to X-MAC, respectively. Duty-cycle length changes with $f_{\text {risk }}$ were also investigated, and $T$ was shortened as forest fire probability increased, ensuring forest fires could be detected faster.

The proposed ADX-MAC protocol for forest fire detection is suitable for various natural disasters. Even if the forest fires are limited, ADX-MAC sensors could be incorporated into existing infrastructure to significantly reduce economic costs.

Author Contributions: Idea and conceptualization: J.-G.K. and D.-W.L.; Methodology: J.-G.K. and J.-W.J.; Software: J.-G.K.; Analysis: J.-G.K.; Visualization, J.-G.K. and D.-W.L.; Project administration: J.-W.J.

Funding: This research was supported by the Ministry of Science and ICT, Korea, under the National Program for Excellence in Software supervised by the Institute for Information \& Communications Technology Promotion (2016-0-00017) and the Basic Science Research Program through the National Research Foundation of Korea funded by the Ministry of Education, Science and Technology (2015R1D1A1A09061368).

Conflicts of Interest: The authors declare no conflicts of interest.

\section{References}

1. Statistics Korea. 2017. Available online: http:/ / kostat.go.kr (accessed on 25 June 2017).

2. International Forest Fire Symposium, Korea National Forest Science Institute. 2016. Available online: http:/ / www.kfri.go.kr (accessed on 25 June 2017).

3. Yang, O.; Heinzelman, W.B. Modeling and Performance Analysis for Duty-cycled MAC Protocols with Applications to S-MAC and X-MAC. IEEE Trans. Mob. Comput. 2012, 11, 905-921. [CrossRef]

4. Lee, J.-H.; Eom, D.-S. A Study of Variable Wakeup Period for Duty-cycled MAC protocol in WSN. J. Inst. Electron. Eng. Korea 2012, 49, 45-55. [CrossRef]

5. Buettner, M.; Yee, G.V.; Anderson, E.; Han, R. X-MAC: A Short Preamble MAC Protocol for Duty-cycled Wireless Sensor Networks. In Proceedings of the 4th International Conference on Embedded Networked Sensor Systems, Boulder, CO, USA, 31 October-3 November 2006; pp. 307-320.

6. Kim, J.-H. Forest Fire Prediction Model Using Weather Data. Master's Thesis, Korea University, Seoul, Korea, 2017.

7. Korea Forest Service. 2017. Available online: http:/ / www.forest.go.kr (accessed on 20 August 2018).

8. Polastre, J.; Hill, J.; Culler, D. Versatile Low Power Media Access for Wireless Sensor Networks. In Proceedings of the 2nd International Conference on Embedded Networked Sensor Systems, Baltimore, MD, USA, 3-5 November 2004; pp. 95-107.

9. Kang, J.-G.; Kim, G.-Y.; Park, S.-H.; Lim, D.-W.; Kang, H.-G.; Jung, J.-W. According to the Traffic Conditions, Duty-cycle Ratio is Changed Hybrid X-MAC Protocol in Networks. In Proceedings of the ITFE Summer Conference, Gangwon, Korea, 1-2 September 2017; pp. 73-74.

10. Weng, C.-E.; Chen, C.-Y. The Performance Study of Optimal Contention Window for IEEE 802.11 DCF Access Control. In Proceedings of the 2012 IEEE 75th Vehicular Technology Conference (VTC Spring), Yokohama, Japan, 6-9 May 2012.

11. Song, N.; Kwak, B.; Song, J.; Miller, L.E. Enhancement of IEEE 802.11 Distributed Coordination Function with Exponential Increase Exponential Decrease Backoff Algorithm. In Proceedings of the IEEE Vehicular Technology Conference (VTC), Jeju, Korea, 22-25 April 2003; Volume 4, pp. 2775-2778.

12. Network Simulator Manual. Available online: http://www.isi.edu/nsam/ns/index.html (accessed on 20 August 2018).

13. Fall, K.; Varadhan, K. The ns Manual (Formerly ns Notes and Documentation); The VINT Project; UC Berkeley: Berkeley, CA, USA, 2003. 